\title{
QUALITY VARIATIONS AND MAXIMAL VARIETY DIFFERENTIATION*
}

\author{
Nicholas ECONOMIDES \\ Columbia University, New York, NY 10027, USA \\ Stanford University, Stanford, CA 94305, USA
}

Received December 1987, final version received November 1988

We allow quality variations in a duopoly of locationally differentiated products a -la-Hotelling (1929). We analyze the impact of quality variations on the choices of the varieties produced. We show that in a sequential game of variety choice and subsequent quality and price choice there exist only maximal variety differentiation equilibria in pure strategies. Maximal variety differentiation is also the perfect pure strategies equilibrium of a sequential game of variety choice followed by quality choice and later by price choice. In both games there is minimal quality differentiation at equilibrium.

\section{Introduction}

The issue of minimal differentiation in locational models has recently stirred a significant amount of research and debate. Much of the debate was prompted by the discovery of D'Aspremont et al. (1979) that Hotelling's (1929) argument was incorrect, and that in his original duopoly the 'principle of minimum differentiation' did not hold. The framework of quick (short-run) price adjustment and slow (long-run) location adjustment has been modelled as a two-stage game with a location stage first and a subsequent price stage. In this framework two questions became focal. First, does a non-cooperative equilibrium in prices exist for all location pairs? Second, do firms have tendencies to move away or towards each other in the location game? As corrected by D'Aspremont et al. (1979), in Hotelling's duopoly with linear transportation costs (disutility of distance in the specification space) there are some price subgames with no equilibria. ${ }^{1}$ Further, when allowed to choose locations non-cooperatively, firms want to move to locations for which equilibrium does not exist in the price subgame. Thus there is no equilibrium in the overall game.

*An earlier version of some of these results appeared as Advertising and Maximal Product Differentiation, Discussion paper no. 316, Department of Economics, Columbia University. Financial support from the National Science Foundation is gratefully acknowledged. I thank two anonymous referees and the editor for helpful suggestions.

${ }^{1}$ This refers to equilibria in pure strategies. 
D'Aspremont et al. (1979) also showed that, with quadratic transportation costs, equilibrium exists for all subgames, and an overall location equilibrium exists with firms at maximal product differentiation. This result did not close the debate for two reasons. First, because the specification of the utility function is arbitrary. Second, because it was not clear if and how the existence of equilibrium was connected with the maximal differentiation property.

A number of variations of Hotelling's model have arisen since, all using the traditional strategic variables (location and price) while changing the specification of the utility function or the underlying space of characteristics. ${ }^{2}$ In this paper we introduce the level of quality as a strategic variable in addition to the price and location strategic variables. ${ }^{3}$ We assess the impact of the new strategic variable on the equilibrium specifications of the products.

We endow firms operating in the standard Hotelling (1929) market for differentiated goods with a technology of quality variation. Quality is defined as a product feature such that, at fixed prices, all consumers desire higher levels of it. In contrast, all consumers do not want 'more' of a feature of variety. We assume that the level of quality ' $a$ ' has no influence on variable production costs, so that the associated costs $C(a)=c a^{2} / 2$ are independent of the level of output. An example of such a quality feature is advertising expenditure, which can be added to any differentiated good. Another example is the speed of calculation of a personal computer. Then the products are computer programs or microchips of quality level ' $a$ ', differentiated in variety (location on Hotelling's 'Main Street') by the task they perform or the extent in which they are graphics-oriented or keyboardoriented. All costs of quality improvement are incorporated in the better design of the program or chip and leave variable production costs unaffected.

Each firm, $i=1,2$, has three strategic variables, price $p_{i}$ quality $a_{i}$, and variety $x_{i}$. We analyze two games. In the first game, varieties are chosen in the first stage, while qualities and prices are chosen in the second stage. In the second game varieties are chosen as before in the first stage, to be followed by qualities' choice in the second stage, and by the choice of prices in the third. In both game structures we seek equilibria in pure strategies.

\footnotetext{
${ }^{2}$ For example, Economides (1986a) showed the existence of equilibria for symmetric locations in a two-dimensional specification space. Economides (1986b) shows the existence of interior (non-maximal differentiation) equilibria when the transportation cost function (disutility of distance) is $f(d)=d^{\alpha}, 5 / 4<\alpha<5 / 3$.

${ }^{3}$ After the first version of this paper was written as Economides (1986c), Neven and Thisse (1987) developed a similar model where consumers are further differentiated with respect to their intensity of preference for quality. Because of this feature, consumers switch gradually to the firm that increases quality, the high-intensity-of-preference consumers being the first to switch. This allows for the added possibility of equilibria where the firms produce quite different qualities but very similar varieties.
} 
Each game structure describes a different market situation. The two-stage game is appropriate for qualities that are as flexible as price in the short run. Advertising is such a quality feature. No design (variety) changes are necessary to change advertising expenditure. In many markets advertising is as flexible as price.

The three-stage game is more appropriate for qualities that are flexible in the medium run but not in the short run. Such a quality is the length of a warranty offered on a product. It cannot be changed as quickly as the price, but it can certainly be changed more quickly than the product design.

Before proceeding with the formal analysis, let us discuss the intuition of the workings of the model. When quality is expensive, very little of it will be used. In this case, we expect to be very close to the original model of Hotelling: pricing is relatively unaffected by location and firms try to come as close as they can to each other to maximize demand. The resulting nonexistence of equilibrium, because of the benefits of undercutting strategies to closely located firms, seems inevitable. When quality improvements are cheaper, it is worthwhile to expend resources to improve the willingness to pay of the captive customers. One expects that the further away a firm moves from its opponent (in the specification spectrum) the more 'insulated' it should become, i.e., the higher its prices (and quality expenditure). The effect on profits of this movement away from the opponent has to be compared with the direct loss in demand a firm may suffer as its product specification may become less appealing to the average consumer. These two effects, 'cultivating' the old customers vs. 'stealing' customers from the opponent, will determine the profitability of moves away from the opponent and the position of the location equilibrium.

\section{The variety $\rightarrow$ (quality and price) game}

For notational convenience we shall use $x$ and $y$ as the variety specifications of products 1 and 2, i.e., $x \equiv x_{1}, y \equiv x_{2}$. Without loss of generality, let $y \geqq x$. The value of product 1 of quality $a_{1}$ sold at price $p_{1}$ to consumer ' $z$ ' is

$$
k+a_{1}-\left[p_{1}+|z-x|\right] .
$$

Thus, quality is normalized so that an increase of one unit in its level pushes up the utility for the product by one unit. $k+a_{1}$ is the highest price a consumer would pay for product $1 .{ }^{4}$ Quality increases of product 1 are valued equally by consumers who differ in their preferences of variety. For example, consumers running different applications value equally increases in

\footnotetext{
${ }^{4}$ The minimal reservation price $k$ is assumed to be sufficiently high so that all consumers always buy a differentiated good.
} 
the speed of a personal computer. ${ }^{5}$ Consumers are identified with the position of the product they like most in the spectrum $[0,1]$. We assume that the distribution of consumers has uniform density $\mu$. The demand for firm 1 is

$$
\begin{aligned}
D_{1} & =\mu & & \text { for } p_{1}-a_{1}<p_{2}-a_{2}-(y-x) \\
& =\mu\left(y+x-p_{1}+p_{2}+a_{1}-a_{2}\right) / 2 & & \text { for }\left|p_{1}-a_{1}-p_{2}+a_{2}\right|<y-x \\
& =0 & & \text { for } p_{1}-a_{1}>p_{2}-a_{2}-(y-x) .
\end{aligned}
$$

The demand for firm 2 is $D_{2}=\mu\left(1-D_{1}\right)$. The revenue functions $R_{i}=p_{i} D_{i}$ consist of a linear and a quadratic part in $p_{i}$ separated by a discontinuity. Assuming zero production costs, ${ }^{6}$ the profit function of firm $i$ is

$$
\Pi_{i}\left(p_{i}, p_{j}, a_{i}, a_{j}, x, y\right)=p_{i} D_{i}-C\left(a_{i}\right)
$$

First-order conditions for profit maximization in prices in the quadratic part of the profit function are solved to derive

$$
p_{1}^{\mathrm{m}}=\left[2+y+x+a_{1}-a_{2}\right] / 3, \quad p_{2}^{\mathrm{m}}=\left[4-(y+x)-a_{1}+a_{2}\right] / 3 .
$$

These are the equilibrium prices for fixed levels of quality, provided that the local maximum we found is a global one. First-order conditions with respect to $a_{i}$ are $^{7}$

$$
p_{i}=2 y a_{i}, i=1,2, \quad \text { where } \quad \lambda=c / \mu \text {. }
$$

Combining these with (1) we derive the equilibrium of the quality-price subgame for locations $(x, y)$ as

$$
\begin{aligned}
& a_{1}^{*}(x, y)=[1 / \lambda+(1-y-x) /(1-3 \lambda)] / 2, \\
& a_{2}^{*}(x, y)=[1 / \lambda-(1-y-x) /(1-3 \lambda)] / 2, \\
& p_{i}^{*}(x, y)=2 \lambda a_{i}^{*}(x, y) .
\end{aligned}
$$

${ }^{5}$ Of course other functional forms could also be assumed depending on the interactions of quality and variety in the utility function. For some products increases in the level of quality of a particular variety are valued higher by those located near that variety and less by others. This will be the case when the feature of quality exhibits a natural synergy with a particular varietal characteristic.

${ }^{6}$ The results can easily be reinterpreted if constant marginal costs are assumed. Then the prices are interpreted as increments of prices above marginal costs. Any convex variable costs will only strengthen the arguments for the existence of equilibrium in the price-quality subgame.

${ }^{7}$ Sufficient conditions for maximization are $\partial^{2} I_{i} / \partial p_{i}^{2}=-\mu<0, \quad \partial^{2} \Pi_{i} / \partial a_{i}^{2}=-c<0$, and $\left(\partial^{2} \Pi_{i} / \partial p_{i}^{2}\right)\left(\partial^{2} \Pi_{i} / \partial a_{i}^{2}\right)-\left(\partial^{2} \Pi_{i} / \partial a_{i} \partial p_{i}\right)^{2}>0 \Leftrightarrow(-\mu)(-c)-(\mu / 2)^{2}>0 \Leftrightarrow \lambda>1 / 4$. 
Note that, when symmetric locations have been selected in the first stage $(x+y=1)$, quality levels and price levels do not differ across firms, i.e., $a_{1}^{*}=a_{2}^{*}, p_{1}^{*}=p_{2}^{*}$. This is a direct consequence of the symmetry of the competitive environment.

Equilibrium profits in the quality-price subgame of locations $(x, y)$ are

$$
\Pi_{i}^{\mathrm{v}}(x, y) \equiv \Pi_{i}\left(p_{i}^{*}, p_{j}^{*}, a_{i}^{*}, a_{j}^{*}, x, y\right)=c\left(a_{i}^{*}(x, y)\right)^{2}(4 \lambda-1) / 2, \quad i=1,2 .
$$

Equilibria in the price-quality subgame exist as long as firms do not find it profitable to 'undercut' the opponent and drive him out of business. An undercutting price-quality combination of firm 1 fulfills $p_{1}^{\mathrm{u}}-a_{1}^{\mathrm{u}} \leqq p_{2}^{*}-a_{2}^{*}-$ $(y-x)$ and results in $D_{1}=\mu$ and $D_{2}=0$. Focusing on symmetric locations $(x+y=1)$, undercutting means

$$
p_{1}^{\mathrm{u}}-a_{1}^{\mathrm{u}} \leqq 2 x-1 /(2 \lambda)
$$

Firm 1 undercuts optimally by choosing $p_{1}^{\mathrm{u}}$ and $a_{1}^{u}$ to maximize $\Pi_{1}^{\mathrm{u}}=$ $\mu p_{1}^{\mathrm{u}}-c\left(a_{1}^{\mathrm{u}}\right)^{2} / 2$ subject to (5). It therefore chooses $a_{1}^{\mathrm{u}}=1 / \lambda p_{1}^{\mathrm{u}}=2 x+1 /(2 \lambda)$ and realizes undercutting profits $\Pi_{1}^{\mathrm{u}}=2 x \mu$. Candidate equilibrium profits are [from (4)], $\Pi_{1}^{*}=c(4 \lambda-1) /\left(8 \lambda^{2}\right)$. Therefore firm 1 will not undercut as long as

$$
\Pi_{1}^{\mathrm{u}} \leqq \Pi_{1}^{*} \Leftrightarrow x \leqq 1 / 4-1 /(16 \lambda) .
$$

For such locations $x$, and the corresponding symmetric locations $y=1-x$, equilibria exist in the price-quality subgame. Fig. 1 shows $E$, the region of existence of Nash equilibria, horizontally shaded in the $x-\lambda$ space.

The equilibrium profits of the quality-price subgame, given by (4), constitute the objective functions of the game of varieties choice. The direction that makes positive the derivative of profits with respect to location defines the 'relocation tendency' for the firm. Here

$$
\mathrm{d} \Pi_{1}^{\mathrm{v}} / \mathrm{d} x=c a_{1}^{*}(4 \lambda-1) \cdot \mathrm{d} a_{1}^{*} / \mathrm{d} x \text { and } \mathrm{d} a_{1}^{*} / \mathrm{d} x=-1 /[2(1-3 \lambda)] \text {. }
$$

Therefore $\mathrm{d} \Pi_{1}^{\mathrm{v}} / \mathrm{d} x<0 \Leftrightarrow(4 \lambda-1)(3 \lambda-1)<0$, which holds for $\lambda \in \boldsymbol{F} \equiv(1 / 4,1 / 3)$. Similarly, $\lambda \in F$ implies $\mathrm{d} \Pi_{2}^{\mathrm{v}} / \mathrm{d} y>0$. Thus, for $\lambda \in F$ firms will go to the edges of the market space in the varieties game, so that maximal differentiation will result, $x^{*}=0, y^{*}=1$. Since these locations are symmetric, the implied prices and quality levels do not differ across firms, $p_{1}^{*}=p_{2}^{*}=1, a_{1}^{*}=a_{2}^{*}=1 /(2 \lambda)$. Thus, we observe maximal varietal differentiation and minimal quality differentiation at equilibrium.

In fig. 1, the area of opposing relocation tendencies is vertically shaded. The horizontal arrow pointing to the left indicates the tendencies of firms to go towards the edge of the market. The double shaded area $\boldsymbol{E} \cap \boldsymbol{F}$ is the 


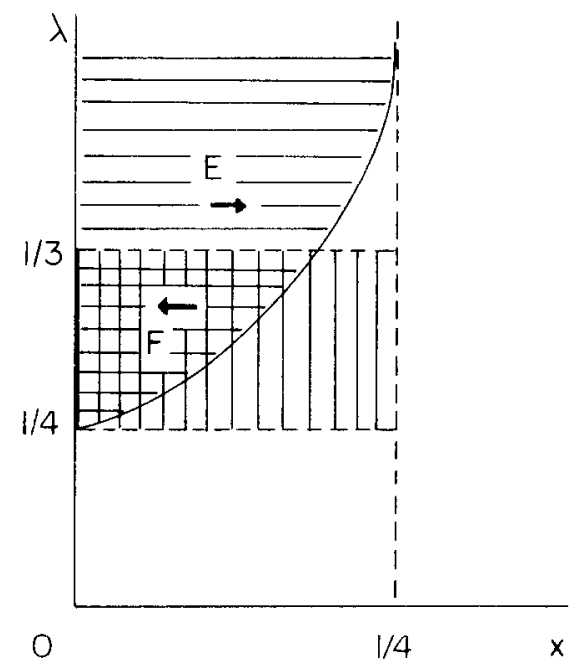

Fig. 1. The region of existence of equilibrium $\boldsymbol{E}$ and the region of relocation tendencies towards the edges of the market, $F$.

locus of cost-location combinations such that an equilibrium exists in the quality-price subgame and relocation tendencies drive firms to the edges of the market. The thick segment on the $\lambda$ axis indicates the resulting equilibrium $x^{*}=0$.

For $\lambda$ outside $F$ we have the following results. $\lambda<1 / 4$ violates positivity conditions. ${ }^{8}$ When costs of quality improvement are high, $\lambda>1 / 3$, relocation tendencies bring firms closer together. Eventually, for close enough locations, undercutting is profitable and firms are driven outside the existence region $E$. Then no equilibrium exists in the quality-price subgame because the firm that is left with zero demand can do better than that by quoting a low positive price. Therefore there are no equilibria in the full game for $\lambda \notin E \cap F$. Thus, there are only maximal differentiation equilibria in the full varietyquality-price game.

Theorem 1. The two-stage game of variety choice in the first stage, and quality and price choice in the second stage, has a unique perfect equilibrium in pure strategies at maximal varietal differentiation, $x^{*}=0, y^{*}=1$, minimal quality differentiation, $a_{1}^{*}=a_{2}^{*}=1 /(2 \lambda)$, and equal prices, $p_{1}^{*}=p_{2}^{*}=1$, for $\lambda \in(1 / 4,1 / 3)$.

To reaffirm our intuition, note that the effect on its own demand of a firm coming closer to its opponent is always positive. This led Hotelling (1929) to

${ }^{8}$ For $\lambda<1 / 4$, second order-conditions fail. Each firm has incentives to simultaneously increase price and quality to infinity. 
claim the 'principle of minimum differentiation.' In the original Hotelling game, with location and price in sequential choice (but with quality levels fixed at zero), prices vary little with location, so that the tendency for minimal differentiation prevails (although minimum differentiation equilibria are not realized because of the superiority of undercutting strategies for closely located firms). In the present game, when costs of quality improvements are low, a movement away from the variety of the opponent increases quality, prices and profits in the subsequent subgame. Higher quality together with slightly higher prices have a positive effect on demand. This effect overshadows the direct negative effect on demand that drove the intuition of minimum varietal differentiation. The availability of low-cost quality improvements creates a tendency towards maximal product differentiation in variety.

\section{The variety $\rightarrow$ quality $\rightarrow$ price game}

In this game variety, quality, and price are chosen sequentially. The price subgame has candidate equilibrium prices given by (1). Substituting them in the profit functions we derive the profit functions of the quality stage,

$$
\begin{aligned}
& \Pi_{i}^{a}\left(a_{i}, a_{j}, x, y\right) \equiv \Pi_{i}\left(p_{i}^{\mathrm{m}}\left(a_{i}, a_{j}\right)\right. \\
& \left.\left.p_{j}^{\mathrm{m}}\left(a_{i}, a_{j}\right), a_{i}, a_{j}, x, y\right)=\mu p_{i}^{\mathrm{m}}\left(a_{i}, a_{j}\right)\right)^{2} / 2-C\left(a_{i}\right) .
\end{aligned}
$$

The common solution of $\partial \Pi_{i}^{a} / \partial a_{i}=0, i=1,2$, defines the equilibrium of the quality stage. ${ }^{9}$

$$
\begin{aligned}
& a_{1}^{* *}=[6 \lambda-2+3 \lambda(y+x)] /[3 \lambda(9 \lambda-2)], \\
& a_{2}^{* *}=[12 \lambda-2-3 \lambda(y+x)] /[3 \lambda(9 \lambda-2)] .
\end{aligned}
$$

Note that, as in the two-stage game, symmetric locations $(x+y=1)$ imply equal quality levels $a_{1}^{* *}=a_{2}^{* *}$ [from (9)] and equal prices across firms as seen by substitution in (1), $p_{1}^{* *}=p_{2}^{* *}$. This comes directly from the symmetry of the game that arises for symmetric locations.

The objective functions of the game in varieties are the equilibrium profits of the quality stage.

$$
\Pi_{i}^{v}(x, y) \equiv \Pi_{i}^{a}\left(a_{i}^{* *}(x, y), a_{j}^{* *}(x, y), x, y\right)=c\left(a_{i}^{* *}\right)^{2}(9 \lambda-1) / 2 .
$$

The relocation tendencies are determined by

\footnotetext{
${ }^{9} \Pi_{i}^{a}$ is concave for $\lambda>1 / 9$.
} 


$$
\mathrm{d} \Pi_{\mathrm{i}}^{\mathrm{v}} / \mathrm{d} x=c a_{i}^{* *}(9 \lambda-1) \cdot \mathrm{d} a_{i}^{* *} / \mathrm{d} x .
$$

Since $\mathrm{d} a_{i}^{* *} / \mathrm{d} x=1 /(9 \lambda-2)$, it follows that

$$
\mathrm{d} \Pi_{1}^{\mathrm{v}} / \mathrm{d} x<0 \Leftrightarrow(9 \lambda-1)(9 \lambda-2)<0,
$$

which holds for $\lambda \in \boldsymbol{G} \equiv(1 / 9,2 / 9)$. Similarly, $\mathrm{d} \Pi_{2}^{\mathrm{v}} / \mathrm{d} y>0$ for $\lambda \in \boldsymbol{G}$. For such $\lambda$ firms will choose the most extreme varieties $x^{* *}=0, y^{* *}=1$. The full equilibrium entails $a_{1}^{* *}=a_{2}^{* *}=1 /(3 \lambda), p_{1}^{* *}=p_{2}^{* *}=1$. In the price subgame played for these locations and quality levels, using an undercutting strategy is undesirable because

$$
p_{1}^{\mathrm{u}} \leqq p_{2}^{* *}-a_{2}^{* *}+a_{1}^{* *}-\left(y^{*}-x^{*}\right)=0 .
$$

Theorem 2. The three-stage game of location choice in the first stage, quality choice in the second stage, and price choice in the third stage, has a unique perfect equilibrium in pure strategies at maximal varietal differentiation $x^{* *}=0$, $y^{* *}=1$, minimal quality differentiation, $a_{1}^{* *}=a_{2}^{* *}=1 /(3 \lambda)$, and equal prices, $p_{1}^{* *}=p_{2}^{* *}=1$, for $\lambda \subset(1 / 9,2 / 9)$.

As in the two-stage game, a movement away from the opponent prompts an increase in the level of quality, driving up prices and profits. This effect is larger than the direct loss in demand the firm suffers as its variety becomes less popular. It implies maximal variety differentiation resulting in symmetric locations at the corners of the interval. The symmetric positions imply a symmetric subgame in qualities and prices which has an equilibrium at minimal quality differentiation.

\section{Conclusions}

We showed that the existence of a technology of quality variation in Hotelling's model of locational duopoly leads to maximally differentiated varieties, both when price and quality are simultaneous strategic variables and when they are sequential strategic variables. Contrary to the intuition of the 'principle of minimum differentiation', firms prefer to maximally differentiate their products in the variety space so that they can achieve higher levels of quality, price and profits. It is striking to see that the simple addition of quality as a strategic variable to the classic model of variety differentiation leads to maximal differentiation, a result directly opposite to the 'Principle of Minimum Differentiation'. At the same time, we observed minimal differentiation in the other two strategic variables, i.e. quality and price. This suggests that it was the absence of enough strategic variables that drove Hotelling's (1929) minimum differentiation tendency in varieties. 


\section{References}

D'Aspremont, Claude, Jean Jaskold-Gabszewicz and Jacques-Francois Thisse, 1979, On Hotelling's 'Stability in competition', Econometrica 47, 1145-1150.

Economides, Nicholas, 1984, The principle of minimum differentiation revisited. European Economic Review 24, no. 3, 345-368.

Economides, Nicholas, 1986a, Nash equilibrium in duopoly with products defined by two characteristics, Rand Journal of Economics 17, no. 3, 431-439.

Economides, Nicholas, 1986b, Minimal and maximal differentiation in Hotelling's duopoly, Economics Letters 21, no. 1, 67-71.

Economides, Nicholas, 1986c, Advertising and maximal product differentiation, Discussion paper no. 316 (Department of Economics, Columbia University, New York, NY).

Hotelling, Harold, 1929, Stability in competition, Economic Journal 39, 41-57.

Neven, Damien and Jacques-Francois Thisse, 1987, Combining horizontal and vertical differentiation: The principle of max-min differentiation, Mimeo. (CORE, Louvain-la-Neuve).

Novshek, William, 1980, Equilibrium in simple spatial (or differentiated product) markets, Journal of Economic Theory 22, no. 2, 313-326.

Salop, Steven, 1979, Monopolistic competition with outside goods, Bell Journal of Economics 10 , no. $1,141-156$. 